\title{
Genetic Risk Score, Combined Lifestyle Factors and Risk of Colorectal Cancer
}

Young Ae Cho, $\mathrm{PhD}, \mathrm{MPH}^{1}$
Jeonghee Lee, MS
Jae Hwan Oh, MD, $\mathrm{PhD}^{2}$
Hee Jin Chang, MD, $\mathrm{PhD}^{2}$
Dae Kyung Sohn, MD, $\mathrm{PhD}^{2}$
Aesun Shin, MD, $\mathrm{PhD}$
Jeongseon Kim, $\mathrm{PhD}^{1}$

${ }^{1}$ Department of Cancer Biomedical Science, Graduate School of Cancer Science and Policy, National Cancer Center, Goyang,

${ }^{2}$ Center for Colorectal Cancer, National Cancer Center Hospital, National Cancer Center, Goyang, ${ }^{3}$ Department of Preventive Medicine, Seoul National University College of Medicine, Seoul, Korea

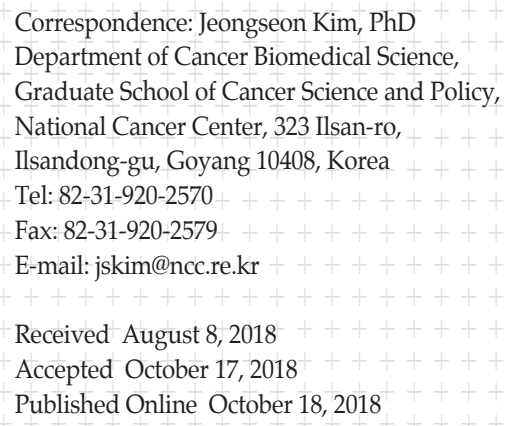

\begin{abstract}
Purpose
Both genetic and lifestyle factors contribute to the risk of colorectal cancer, but each individual factor has a limited effect. Therefore, we investigated the association between colorectal cancer and the combined effects of genetic factors or/and lifestyle risk factors.
\end{abstract}

\section{Materials and Methods}

In a case-control study of 632 colorectal cancer patients and 1,295 healthy controls, we quantified the genetic risk score for colorectal cancer using 13 polymorphisms. Furthermore, we determined a combined lifestyle risk score including obesity, physical activity, smoking, alcohol consumption, and dietary inflammatory index. The associations between colorectal cancer and risk score using these factors were examined using a logistic regression model.

\section{Results}

Higher genetic risk scores were associated with an increased risk of colorectal cancer (odds ratio [OR], 2.57; 95\% confidence interval [Cl], 1.89 to 3.49 for the highest tertile vs. lowest tertile). Among the modifiable factors, previous body mass index, physical inactivity, heavy alcohol consumption, and a high inflammatory diet were associated with an increased risk of colorectal cancer. A higher lifestyle risk score was associated with an increased risk of colorectal cancer (OR, 5.82; $95 \% \mathrm{Cl}, 4.02$ to 8.44 for the highest tertile vs. lowest tertile). This association was similar in each genetic risk category.

\section{Conclusion}

Adherence to a healthy lifestyle is associated with a substantially reduced risk of colorectal cancer regardless of individuals' genetic risk.

\section{Introduction}

Colorectal cancer is the third most common cancer in men and the second most common cancer in women worldwide [1]. A family history of colorectal cancer is an independent risk factor, suggesting that inherited DNA sequence variants contribute to the causation of colorectal cancer [2]. Recently, several genome-wide association studies have identified polymorphisms associated with colorectal cancer risk [3-6].
Key words

Colorectal cancer, Genes, Lifestyle, Risk score 
large international variation in the incidence of colorectal cancer [9]. Several previous studies have used a genetic risk score to predict individuals' predetermined risk $[3,10]$. Additionally, some previous studies have examined the association between combined lifestyle factors and the incidence of colorectal cancer [11-14]. However, these studies are not comparable due to the use of different lifestyle factors, different cut-off points and different scoring systems [11]. From a public health point of view, the incidence of colorectal cancer may be reduced by modifying lifestyle risk factors and screening individuals at a high risk. However, few studies have examined the association between combined genetic and lifestyle factors and the risk of developing colorectal cancer.

Thus, we constructed a genetic risk score using validated common colorectal cancer susceptibility loci identified from genome-wide association studies. In addition, we aimed to identify modifiable lifestyle factors and develop a combined risk score, and determine whether lifestyle factors are affected differently by un-modifiable risk factors, such as genetic factors.

\section{Materials and Methods}

\section{Study populations}

This study included cases of newly diagnosed colorectal cancer patients at the Center for Colorectal Cancer of the National Cancer Center, Korea, between August 2010 and August 2013. Of the 925 patients who agreed to participate in the study and completed the questionnaires, two patients were excluded due to implausible energy intakes. In total, 923 patients were included in the analysis. The control participants were recruited between October 2007 and December 2014 among individuals visiting the Center for Cancer Prevention and Detection at the same hospital for a health check-up program provided by the National Health Insurance Cooperation, which covers the entire Korean population. Of the 9,157 healthy subjects who agreed to participate in the study and completed the questionnaires, 120 subjects were excluded due to the implausible energy intake. Of the remaining 9,037 individuals, two controls per case were randomly selected and frequency-matched by gender and 5-year age group. Additionally, individuals with missing blood samples and genotyping data were excluded. Ultimately, 632 colorectal cancer patients and 1,295 healthy controls were included in the final analysis (S1 Fig.).

\section{Assessment of modifiable lifestyle risk factors}

Information regarding the participants' demographic and lifestyle risk factors (e.g., smoking, alcohol consumption, and regular exercise) was collected by in-person interviews or structured questionnaires at initial recruitment, prior to cancer diagnosis. The dietary intake was assessed using a validated 106-item semi-quantitative food frequency questionnaire specifically developed for this population encompassing commonly consumed food items. The validity and reproducibility of the questionnaire have been previously reported [15]. Each participant provided their average frequency of eating and typical portion sizes in the year preceding the interview. These values were converted to obtain daily nutrient intake values using a scale with nine frequency categories and three portion size categories. We calculated the dietary inflammatory index (DII), and the calculation of the DII has been previously described [16,17]. In the present study, 35 food components were included to calculate DII: protein, fat, carbohydrate, fiber, monounsaturated fatty acid, saturated fatty acid, polyunsaturated fatty acid, n-3 fatty acid, n-6 fatty acid, cholesterol, thiamin, riboflavin, niacin, vitamin $B_{6}$, vitamin $B_{12}$, vitamin $C$, folic acid, vitamin $A$, vitamin $D$, vitamin $E, \beta$-carotene, iron, magnesium, selenium, zinc, garlic, ginger, onion, green tea, flavan-3-ol, flavone, flavonol, flavanone, anthocyanidin, and isoflavone.

\section{Single-nucleotide polymorphism selection and genotyp- ing}

The single-nucleotide polymorphisms (SNPs) were selected as previously described [18]. Briefly, 30 SNPs were selected from the literature based on their association with colorectal cancer in previous genome-wide association studies [18]. Details regarding the selected SNPs are provided in S2 Table.

The genotyping was conducted as described below. Genomic DNA was extracted using the MagAttract DNA Blood M48 Kit (Qiagen, Hilden, Germany) and BioRobot M48 automatic extraction equipment (Qiagen) according to the manufacturer's instructions. The genotyping was performed using a MassARRAY iPLEX Gold Assay (Agena Bioscience, San Diego, CA). To control the genotyping quality, duplicate samples of $3 \%$ of the subjects were included in our initial genotyping analysis; the rate of discordance was $<1 \%$.

\section{Construction of the unweighted and weighted genetic risk score}

To construct the genetic risk score, the independent association between 30 SNPs and colorectal cancer was initially examined using a logistic regression under allele-based 
model. Among them, 13 SNPs were significantly associated with the risk of colorectal cancer in the present study. Therefore, these SNPs were used to calculate the genetic risk scores. We constructed both unweighted and weighted genetic risk scores [19]. The unweighted genetic risk scores of individual participants were created by adding the number of risk alleles (i.e., 0,1 , or 2 ) at each SNP. Additionally, we calculated the weighted genetic risk score by multiplying by the effect size (the SNP's $\beta$ coefficient from the current logistic regression model) in the current case-control study. The 13 products were then summed to create a weighted genetic risk score for each study participant.

\section{Development of lifestyle risk score}

We generated a modifiable lifestyle index based on a priori knowledge of risk factors for colorectal cancer and public health recommendations. The lifestyle factors were characterized as harmful based on their independent association with colorectal cancer. First, both current and previous (prior 2 years) body mass indexes (BMIs) were calculated because the current BMI could be affected by the disease status in the case-control studies [20]. The BMI was categorized according to the World Health Organization criteria for the Asian population $\left(<25 \mathrm{~kg} / \mathrm{m}^{2}\right.$ vs. $\left.\geq 25 \mathrm{~kg} / \mathrm{m}^{2}\right)$. We compared the current and prior BMI, and found that the prior BMI is more significantly associated with colorectal cancer in the present study. Therefore, the prior BMI was used as an index for obesity. Second, physical activity was defined as conducting exercise regularly. Third, an unhealthy diet was defined based on the level of DII. Diet was classified as a low or high inflammatory diet based on the median intake levels of DII scores in the control group. Fourth, cigarette smoking was classified as ever or never. Smoking was chosen based on previous evidence and public health recommendations $[7,21]$. Fifth, people who drank more than $30 \mathrm{~g}$ of alcohol/ day were considered heavy drinkers based on the association between alcohol consumption and colorectal cancer risk in the current study.

Finally, we assigned a lifestyle risk score ( 0 for healthy and 1 for unhealthy) to each participant and summed the scores of the five lifestyle factors; consequently, the lifestyle index ranged from zero (healthiest) to five (least healthy) points [11].

\section{Statistical analysis}

The differences in the demographic and lifestyle factors between the cases and controls were analyzed using the chisquaqre test for categorical variables and Student's $\mathrm{t}$ test for continuous variables.

The association between the risk factors (genetic and life- style risk factors) and colorectal cancer risk was analyzed using unconditional logistic regression models. The genetic risk score was calculated using 13 SNPs and divided into tertiles based on scores among controls: low (20\%-30\%), intermediate (50\%-60\%), and high (20\%-30\%). Because some of participants had the same risk scores, we could not divide subjects into the same proportion. The combined lifestyle factors were also divided into tertiles: low (defined as no or only one lifestyle risk factor), intermediate (defined as 2 or 3 lifestyle risk factors), and high (defined as four or five lifestyle risk factors). The lowest genetic or lifestyle risk factors were considered references. The multivariable model was adjusted for age, sex, family history of colorectal cancer, and education and additionally adjusted for the five modifiable risk factors (i.e., prior BMI, physical activity, DII, smoking, and alcohol consumption) as needed. Furthermore, we conducted a stratified analysis of the association between the lifestyle risk factors and colorectal cancer risk according to the genetic risk category. We used the results based on the unweighted and weighted risk score in further analyses. A multinomial logistic regression model was used in the analyses stratified by anatomical location (i.e., colon and rectal cancer). Interactions between genetic risk scores and lifestyle risk scores were assessed using the likelihood ratio test by comparing the model with the interaction term, with the model containing only main effects.

All statistical analyses were performed using SAS 9.2 (SAS Institute Inc., Cary, NC) and STATA ver. 14 software (Stata Corp., College Station, TX). A two-sided p-value of less than 0.05 indicated statistical significance.

\section{Ethical statement}

All participants provided written informed consent, and the study protocol was approved by the Institutional Review Board of the National Cancer Center (IRB No. NCCNCS-10350 and NCC2015-0202).

\section{Results}

Table 1 shows the general characteristics of the study populations. The colorectal cancer patients had a higher family history ( $\mathrm{p}=0.029)$, had a low education level $(\mathrm{p}<0.001)$, were unlikely to perform regular exercise $(\mathrm{p}<0.001)$, and had a higher energy intake $(\mathrm{p}<0.001)$ and higher DII $(\mathrm{p}<0.001)$. However, no differences were observed in age, sex, smoking, and alcohol intake. The cases did not differ from the controls in their current BMI but had a higher prior BMI $(\mathrm{p}<0.001)$.

The association between colorectal cancer and selected 
Table 1. General characteristics of the study populations

\begin{tabular}{|c|c|c|c|}
\hline & Control $(n=1,295)$ & Case $(n=632)$ & p-value \\
\hline Age (yr) & $56.0 \pm 5.1$ & $56.4 \pm 9.6$ & 0.380 \\
\hline \multicolumn{4}{|l|}{ Sex } \\
\hline Female & $408(31.5)$ & $203(32.1)$ & 0.786 \\
\hline Male & $887(68.5)$ & $429(67.9)$ & \\
\hline Family history (yes)a) & $71(5.5)$ & $51(8.1)$ & 0.029 \\
\hline \multicolumn{4}{|l|}{ Education (yr) } \\
\hline$<12$ & $605(47.1)$ & $472(74.7)$ & $<0.001$ \\
\hline$\geq 12$ & $679(52.9)$ & $160(25.3)$ & \\
\hline \multicolumn{4}{|l|}{ BMI $\left(\mathrm{kg} / \mathrm{m}^{2}\right)$} \\
\hline$<25$ & $862(66.6)$ & $431(68.2)$ & 0.474 \\
\hline$\geq 25$ & $433(33.4)$ & $201(31.8)$ & \\
\hline \multicolumn{4}{|l|}{ Prior BMI $\left(\mathrm{kg} / \mathrm{m}^{2}\right)^{\mathrm{b})}$} \\
\hline$<25$ & $827(63.9)$ & $322(51.0)$ & $<0.001$ \\
\hline$\geq 25$ & $468(36.1)$ & $310(49.1)$ & \\
\hline Physical activity (yes) & $771(59.8)$ & $207(32.8)$ & $<0.001$ \\
\hline \multicolumn{4}{|l|}{ Smoking } \\
\hline Never & $565(43.6)$ & $286(45.3)$ & 0.500 \\
\hline Ever & $730(56.4)$ & $346(54.8)$ & \\
\hline \multicolumn{4}{|l|}{ Alcohol consumption } \\
\hline Never & $380(29.3)$ & $192(30.4)$ & 0.640 \\
\hline Ever & $915(70.7)$ & $440(69.6)$ & \\
\hline Total calorie intake (kcal) & $1,696.3 \pm 561.6$ & $2,023.0 \pm 529.2$ & $<0.001$ \\
\hline DII & $0.94 \pm 2.24$ & $1.77 \pm 1.97$ & $<0.001$ \\
\hline
\end{tabular}

Values are presented as mean \pm standard deviation or number (\%). BMI, body mass index; DII, dietary inflammatory index.

a)First-degree relative, b)BMI from 2 years ago.

Table 2. Association between genetic risk score and colorectal cancer risk, stratified by anatomical site ${ }^{\text {a) }}$

\begin{tabular}{|c|c|c|c|c|c|c|c|}
\hline \multirow{2}{*}{$\begin{array}{l}\text { Genetic } \\
\text { risk sore }\end{array}$} & \multirow{2}{*}{$\begin{array}{c}\text { No. of } \\
\text { controls }(\%)\end{array}$} & \multicolumn{2}{|c|}{ Colorectal cancer } & \multicolumn{2}{|c|}{ Colon cancer } & \multicolumn{2}{|c|}{ Rectal cancer } \\
\hline & & No. $(\%)$ & OR $(95 \% \mathrm{CI})^{\mathrm{b})}$ & No. $(\%)$ & OR $(95 \% \mathrm{CI})^{\mathrm{b})}$ & No. $(\%)$ & OR $(95 \% \mathrm{CI})^{\mathrm{b})}$ \\
\hline \multicolumn{8}{|c|}{ Unweighted } \\
\hline$\leq 11$ & $273(21.1)$ & 80 (12.7) & 1.0 (reference) & $43(13.5)$ & 1.0 (reference) & $37(12.2)$ & 1.0 (reference) \\
\hline $12-16$ & $742(57.3)$ & $341(54.0)$ & $1.63(1.20-2.21)$ & $165(51.9)$ & $1.44(0.98-2.10)$ & $168(55.3)$ & $1.77(1.18-2.64)$ \\
\hline$\geq 17$ & $280(21.6)$ & $211(33.4)$ & $2.87(2.05-4.01)$ & $110(34.6)$ & $2.71(1.80-4.09)$ & $99(32.6)$ & $2.99(1.93-4.63)$ \\
\hline \multicolumn{8}{|c|}{ Weighted } \\
\hline $\mathrm{T} 1$ & $323(24.9)$ & 98 (15.5) & 1.0 (reference) & $52(16.4)$ & 1.0 (reference) & $46(15.1)$ & 1.0 (reference) \\
\hline $\mathrm{T} 2$ & $647(50.0)$ & $292(46.2)$ & $1.45(1.09-1.94)$ & $141(44.3)$ & $1.30(0.91-1.87)$ & $146(48.1)$ & $1.57(1.08-2.28)$ \\
\hline $\mathrm{T} 3$ & $352(25.1)$ & $242(38.3)$ & $2.57(1.89-3.49)$ & $125(39.3)$ & $2.46(1.69-3.60)$ & $112(36.8)$ & 2.59 (1.74-3.85) \\
\hline
\end{tabular}

$\mathrm{CI}$, confidence interval; OR, odds ratio; T, tertile; SNP, single nucleotide polymorphism. ${ }^{\text {a) }}$ Genetic risk score was calculated using 13 SNPs, b) Adjusted for age, sex, family history of colorectal cancer, education, obesity, physical activity, smoking, alcohol consumption, and dietary inflammatory index.

SNPs among the 30 investigated polymorphisms was examined; only 13 SNPs were significantly associated with colorectal cancer (S3 Table). Several SNPs showed slightly different associations between the colon cancer and rectal cancer patients.

Table 2 presents the association between the genetic risk score and the risk of colorectal cancer. In this study, the genetic risk scores were approximately normally distributed. The 
Table 3. Association between modifiable risk factor and colorectal cancer risk, stratified by anatomical location

\begin{tabular}{|c|c|c|c|c|c|c|c|}
\hline & \multirow{2}{*}{$\begin{array}{c}\text { No. of } \\
\text { controls }(\%)\end{array}$} & \multicolumn{2}{|c|}{ Colorectal cancer } & \multicolumn{2}{|c|}{ Colon cancer } & \multicolumn{2}{|c|}{ Rectal cancer } \\
\hline & & No. $(\%)$ & OR $(95 \% \text { CI) })^{a}$ & No. $(\%)$ & OR $(95 \% \text { CI })^{\mathrm{a})}$ & No. $(\%)$ & OR $(95 \% \mathrm{CI})^{\mathrm{a})}$ \\
\hline \multicolumn{8}{|c|}{ Prior BMI $\left(\mathrm{kg} / \mathrm{m}^{2}\right)^{\mathrm{b})}$} \\
\hline$<25$ & $827(63.9)$ & $322(51.0)$ & 1.0 (reference) & $146(45.9)$ & 1.0 (reference) & $166(54.6)$ & 1.0 (reference) \\
\hline$\geq 25$ & $468(36.1)$ & $310(49.1)$ & $1.76(1.43-2.18)$ & $172(54.1)$ & $2.20(1.69-2.87)$ & $138(45.4)$ & $1.47(1.12-1.93)$ \\
\hline \multicolumn{8}{|c|}{ Physical activity } \\
\hline Yes & $771(59.8)$ & $207(32.8)$ & 1.0 (reference) & $117(36.8)$ & 1.0 (reference) & $88(29.0)$ & 1.0 (reference) \\
\hline No & $518(40.2)$ & $425(67.3)$ & $2.71(2.19-3.36)$ & $201(63.2)$ & $2.26(1.73-2.95)$ & $216(71.1)$ & $3.25(2.45-4.31)$ \\
\hline \multicolumn{8}{|l|}{ Smoking } \\
\hline Never & $565(43.6)$ & $286(45.3)$ & 1.0 (reference) & 155 (48.7) & 1.0 (reference) & $125(41.1)$ & 1.0 (reference) \\
\hline Ever & $730(56.4)$ & $346(54.8)$ & $0.74(0.55-0.98)$ & $163(51.3)$ & $0.70(0.49-0.99)$ & $179(58.9)$ & $0.79(0.55-1.14)$ \\
\hline \multicolumn{8}{|c|}{ Alcohol (g/day) } \\
\hline$\leq 30$ & $1,154(89.1)$ & $501(79.3)$ & 1.0 (reference) & $258(81.1)$ & 1.0 (reference) & $234(77.0)$ & 1.0 (reference) \\
\hline$>30$ & 141 (10.9) & $131(20.7)$ & $1.88(1.40-2.53)$ & $60(18.9)$ & $1.76(1.22-2.55)$ & $70(23.0)$ & $2.02(1.41-2.89)$ \\
\hline \multicolumn{8}{|l|}{ DII } \\
\hline Low & $647(50.0)$ & $242(38.3)$ & 1.0 (reference) & $125(39.3)$ & 1.0 (reference) & $114(37.5)$ & 1.0 (reference) \\
\hline High & $648(50.0)$ & $390(61.7)$ & $1.38(1.12-1.71)$ & $193(60.7)$ & $1.39(1.06-1.81)$ & $190(62.5)$ & $1.37(1.04-1.80)$ \\
\hline \multicolumn{8}{|c|}{ Lifestyle risk score ${ }^{c)}$} \\
\hline 0 or 1 & $458(35.5)$ & $121(19.2)$ & 1.0 (reference) & $60(18.9)$ & 1.0 (reference) & 57 (18.9) & 1.0 (reference) \\
\hline 2 or 3 & $727(56.4)$ & $376(59.5)$ & $2.24(1.72-2.91)$ & $195(61.3)$ & $2.51(1.79-2.53)$ & $176(57.9)$ & $2.08(1.47-2.94)$ \\
\hline 4 or 5 & $104(8.1)$ & $135(21.4)$ & $5.82(4.02-8.44)$ & $63(19.8)$ & $6.23(3.89-9.96)$ & $71(23.4)$ & $5.72(3.60-9.08)$ \\
\hline
\end{tabular}

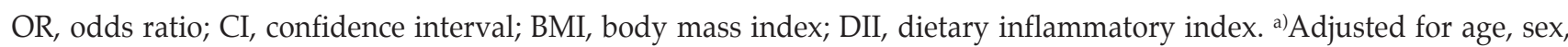
family history of colorectal cancer, and education. Additionally adjusted for prior BMI, physical activity, smoking, alcohol, and DII, if applicable, b)BMI from 2 years ago, c)Scored by summing the prior BMI (2 years ago), physical activity, smoking, alcohol consumption, and DII.

unweighted and weighted genetic risk scores ranged between 5 and 23 (mean \pm SD, 14.40 \pm 3.17 ) and between 0.93 and $4.63($ mean \pm SD, $2.92 \pm 0.61)$, respectively. Compared to the lowest tertile, those in the highest tertile had a higher risk of colorectal cancer according to both the unweighted (odds ratio [OR], 2.87; 95\% confidence interval [CI], 2.05 to 4.01 for the highest vs. lowest tertile) and weighted (OR, 2.57; 95\% CI, 1.89 to 3.49 for the highest vs. lowest tertile) genetic risk scores. After stratifying by anatomical site, these associations were slightly stronger among patients with rectal cancer than those with colon cancer. Additionally, after stratifying by age, slightly stronger association was observed among younger people $(<50$ years) than older people ( $\geq 50$ years) (S4 Table). The performance of the genetic risk score using the weighted genetic risk score was similar to that using a simple count of risk alleles.

Table 3 presents the association between the modifiable risk factors and the colorectal cancer risk. Among the investigated lifestyle factors, the prior BMI, physical inactivity, heavy alcohol consumption, and high DII score were associated with an increased risk of colorectal cancer. All factors combined resulted in a lifestyle risk score that was highly associated with an increased risk of colorectal cancer (OR, $5.82 ; 95 \%$ CI, 4.02 to 8.44 for the highest vs. lowest tertile). After stratifying the data by anatomical site, the association observed among the colon cancer patients (OR, 6.23; 95\% CI, 3.89 to 9.96 for the highest vs. lowest risk score) was slightly stronger than that among the rectal cancer patients (OR, 5.72; 95\% CI, 3.60 to 9.08 for the highest vs. lowest risk score).

The combined effect of the genetic risk score and lifestyle risk score on the risk of colorectal cancer is shown in Table 4. Both the genetic risk scores and the lifestyle risk scores were divided into tertiles. As the genetic risk score increased into the highest tertile, the colorectal cancer risk increased. Additionally as the lifestyle risk score increased into the highest tertile, the colorectal cancer risk increased even more. In the analysis using unweighted genetic risk score (GRS), the participants in the highest tertile for both the genetic risk score and lifestyle risk score had an approximately 12 times higher risk of colorectal cancer than those in the lowest tertile for both the genetic risk score and lifestyle risk score (OR, 12.57; 95\% CI, 5.77 to 27.39). This association seems to be stronger among the rectal cancer patients (OR, 17.71; 95\% CI, 5.80 to $54.04)$ than that among the colon cancer patients (OR, 10.34; 
Table 4. Association between colorectal cancer risk and combined risk score of genetic and lifestyle risk ${ }^{\text {a) }}$

\begin{tabular}{|c|c|c|c|}
\hline \multirow{2}{*}{ Lifestyle risk score } & \multicolumn{3}{|c|}{ Genetic risk score $^{\text {b) }}$} \\
\hline & Low & Intermediate & High \\
\hline \multicolumn{4}{|c|}{ Unweighted genetic risk score } \\
\hline \multicolumn{4}{|c|}{ Colorectal cancer } \\
\hline Low & 1.0 (reference) & $1.32(0.72-2.42)$ & $2.81(1.45-5.45)$ \\
\hline Intermediate & $1.78(0.93-3.42)$ & $3.30(1.86-5.86)$ & $5.42(2.98-9.88)$ \\
\hline High & $6.29(2.78-14.21)$ & $8.67(4.45-16.90)$ & $12.57(5.77-27.39)$ \\
\hline \multicolumn{4}{|l|}{ Colon cancer } \\
\hline Low & 1.0 (reference) & $1.00(0.48-2.07)$ & $1.50(0.65-3.46)$ \\
\hline Intermediate & $1.33(0.60-2.95)$ & $2.49(1.26-4.93)$ & $4.79(2.37-9.69)$ \\
\hline High & $5.30(2.01-13.98)$ & $6.11(2.74-13.64)$ & $10.34(4.16-25.71)$ \\
\hline \multicolumn{4}{|l|}{ Rectal cancer } \\
\hline Low & 1.0 (reference) & $1.83(0.68-4.91)$ & $5.49(1.99-15.12)$ \\
\hline Intermediate & $2.76(0.99-7.68)$ & $4.86(1.90-12.43)$ & $6.90(2.63-18.11)$ \\
\hline High & $8.63(2.66-27.99)$ & $13.71(4.97-37.83)$ & $17.71(5.80-54.04)$ \\
\hline \multicolumn{4}{|c|}{ Weighted genetic risk score } \\
\hline \multicolumn{4}{|c|}{ Colorectal cancer } \\
\hline Low & 1.0 (reference) & $1.17(0.66-2.07)$ & $2.75(1.51-5.03)$ \\
\hline Intermediate & $1.86(1.03-3.33)$ & $3.12(1.84-5.29)$ & $4.95(2.88-8.52)$ \\
\hline High & $6.26(2.94-13.33)$ & $8.15(4.29-15.48)$ & $11.40(5.63-23.09)$ \\
\hline \multicolumn{4}{|l|}{ Colon cancer } \\
\hline Low & 1.0 (reference) & $0.93(0.46-1.89)$ & $1.62(0.75-3.49)$ \\
\hline Intermediate & $1.44(0.69-2.98)$ & $2.47(1.30-4.69)$ & $4.57(2.38-8.77)$ \\
\hline High & $5.70(2.31-14.07)$ & $5.99(2.73-13.15)$ & $9.49(4.10-21.97)$ \\
\hline \multicolumn{4}{|l|}{ Rectal cancer } \\
\hline Low & 1.0 (reference) & $1.47(0.61-3.57)$ & $4.53(1.89-10.96)$ \\
\hline Intermediate & $2.61(1.08-6.29)$ & 4.15 (1.84-9.37) & $5.63(2.45-12.94)$ \\
\hline High & $7.49(2.61-21.47)$ & $11.89(4.79-59.54)$ & $14.46(2.46-38.30)$ \\
\hline
\end{tabular}

a) Adjusted for age, sex, family history of colorectal cancer, and education, b) Both weighted and unweighted genetic risk score were used.

95\% CI, 4.16 to 25.71 ). The analysis using weighted GRS also shows similar associations. The association was similar in each genetic category.

\section{Discussion}

In the present study, the inherited genetic variation and lifestyle factors contributed independently to the susceptibility to colorectal cancer. According to the stratified analysis by genetic risk, the modifiable lifestyle risk factors were associated with similar risk increases in colorectal cancer risk across each stratum of genetic risk. The combined effects of genetics and lifestyle on the risk of colorectal cancer were stronger in the rectal cancer patients than those in the colon cancer patients.
The contribution of an inherited genetic predisposition to the causation of sporadic colorectal cancer remains unclear. In twin studies using combined data from three Nordic countries, colorectal cancer was shown to be relatively more heritable than other cancer types [2]. Recently, genome-wide association studies have identified several common genetic markers that are significantly associated with colorectal cancer [3-6]. Although the predicted risk conferred by each individual polymorphism tends to be modest, together, these polymorphisms have cumulative effects on colorectal cancer risk $[2,22]$. Therefore, several studies used a combined genetic risk score to elucidate the effect of heritability on colorectal cancer [3,10]. In a case-cohort study, Jung et al. [3] reported that participants in the highest quartiles of the genetic risk score had an elevated risk of colorectal cancer (hazard ratio, 2.65; 95\% CI, 1.43 to 4.91) compared with those in the lowest quartile using seven SNPs. In the present study, we used 13 SNPs to calculate the genetic risk score and found a 
cumulatively increased risk of colorectal cancer among those carrying multiple risk alleles. In addition, the estimated hereditary components in the younger participants were slightly higher than those in the older participants; this finding is consistent with observations in which the hereditary effects were the strongest in patients with early-onset cancers $[2,18]$. According to the present study, calculated genetic risk score might be useful in identifying high-risk subjects carrying multiple risk alleles [22].

Lifestyle plays a principal role in causing colorectal cancer. In the present study, heavy alcohol consumption, obesity, physical inactivity, and an unhealthy diet were independent risk factors for colorectal cancer, and the combined risk score of the five risk factors was strongly associated with the risk of colorectal cancer. Several previous studies have examined the association between combined lifestyle factors and the incidence of colorectal cancer [11-14]. In a Danish cohort study, individuals with an increasing number of healthy lifestyle factors (i.e., physical activity, smoking status, alcohol consumption, certain dietary components, and waist circumference) experienced a stepwise decrease in the risk of colon cancer during the follow-up period [11]. In the Healthy Professional Follow-up study, a risk score composed of BMI, physical inactivity, alcohol consumption, smoking habits, red meat consumption, and folic acid supplement use showed that a poorer lifestyle was associated with an increased risk of developing colon cancer [12]. In the present study, we selected lifestyle factors and cut-offs based on the literature and our data, and suggested that the risk of colorectal cancer can be reduced by changing lifestyles [23]. Even though the influence of many risk factors on colorectal cancer may be very complex depending on age and other exposures, this simple score may guide to the general population to modify their lifestyle.

The exact mechanism by which the combined lifestyle and genetic factors affect the risk for colorectal cancer is unknown. The plausible biological effects of each factor are pleiotropic in nature and likely involve overlapping influence on pathways that are relevant to colorectal cancer development. Obesity, physical activity, and an unhealthy diet may contribute to metabolic syndrome, insulin resistance, modification of hormonal levels, immune function, inflammation, gastrointestinal transit time, and bile acid metabolism [16,24]. Cigarettes contain more than 7,000 dangerous chemicals comprising different carcinogens and cigarette smoking also increases inflammation [25]. However, the effects of these lifestyle factors may differ based on individuals' genetic susceptibility because certain gene could be expressed only when activated by environmental factors [23]. Therefore, gene-environment interactions have a significant influence on the susceptibility to colorectal cancer. Furthermore, colorectal cancer is a heterogeneous disease. The colon and rec- tum serve different physiological functions and have different enzyme activities, fecal composition, bile acid metabolism, and intestinal transit time [26,27]. Therefore, colorectal cancer has different genetic and environmental risk factors depending on the anatomical location and different molecular pathways of carcinogenesis [28,29]. In the present study, the higher genetic and lifestyle risk groups seems to be at a higher risk of developing rectal cancer than developing colon cancer.

Risk stratification of target populations using simple questionnaires or genetic testing may be helpful to identify individuals who should undergo colorectal cancer screening [30]. In addition, public health interventions must be conducted to modify lifestyles, particularly for those with a high genetic risk. The findings of the present study may help to provide strategy for preventing colorectal cancer. However, the findings of the current study should be interpreted with caution because of the following limitations. First, this is a case-control study and thus may contain potential selection and information bias. Controls who seek a screening center may be more health-conscious and have healthier lifestyle than the general population. Second, a certain degree of measurement error and misclassification of exposure to risk factors is inevitable. Third, the results of the stratified analyses may be more attenuated due to relatively small sample size [18].

In conclusion, adherence to a healthy lifestyle is associated with a substantially reduced risk of colorectal cancer regardless of individuals' genetic risk, suggesting that genetic risk might be attenuated by a favorable lifestyle. Our study highlights the useful public health message that even modest differences in lifestyle might have a substantial impact on colorectal cancer risk and emphasizes the importance of convincing people to follow lifestyle recommendations.

\section{Electronic Supplementary Material}

Supplementary materials are available at Cancer Research and Treatment website (https: // www.e-crt.org).

\section{Conflicts of Interest}

Conflict of interest relevant to this article was not reported.

\section{Acknowledgments}

This work was supported by grants from the National Cancer Center in Korea (1710882, 1810090), the National Research Foundation of Korea (2015R1C1A2A01053728). 


\section{References}

1. Torre LA, Bray F, Siegel RL, Ferlay J, Lortet-Tieulent J, Jemal A. Global cancer statistics, 2012. CA Cancer J Clin. 2015;65:87108.

2. Lichtenstein P, Holm NV, Verkasalo PK, Iliadou A, Kaprio J, Koskenvuo M, et al. Environmental and heritable factors in the causation of cancer: analyses of cohorts of twins from Sweden, Denmark, and Finland. N Engl J Med. 2000;343:78-85.

3. Jung KJ, Won D, Jeon C, Kim S, Kim TI, Jee SH, et al. A colorectal cancer prediction model using traditional and genetic risk scores in Koreans. BMC Genet. 2015;16:49.

4. Zhang B, Jia WH, Matsuda K, Kweon SS, Matsuo K, Xiang YB, et al. Large-scale genetic study in East Asians identifies six new loci associated with colorectal cancer risk. Nat Genet. 2014;46:533-42.

5. Jia WH, Zhang B, Matsuo K, Shin A, Xiang YB, Jee SH, et al. Genome-wide association analyses in East Asians identify new susceptibility loci for colorectal cancer. Nat Genet. 2013;45: 191-6.

6. Houlston RS, Cheadle J, Dobbins SE, Tenesa A, Jones AM, Howarth K, et al. Meta-analysis of three genome-wide association studies identifies susceptibility loci for colorectal cancer at 1q41, 3q26.2, 12q13.13 and 20q13.33. Nat Genet. 2010;42: 973-7.

7. Huxley RR, Ansary-Moghaddam A, Clifton P, Czernichow S, Parr CL, Woodward M. The impact of dietary and lifestyle risk factors on risk of colorectal cancer: a quantitative overview of the epidemiological evidence. Int J Cancer. 2009;125:171-80.

8. Center MM, Jemal A, Smith RA, Ward E. Worldwide variations in colorectal cancer. CA Cancer J Clin. 2009;59:366-78.

9. Aran V, Victorino AP, Thuler LC, Ferreira CG. Colorectal cancer: epidemiology, disease mechanisms and interventions to reduce onset and mortality. Clin Colorectal Cancer. 2016;15: 195-203.

10. Iwasaki M, Tanaka-Mizuno S, Kuchiba A, Yamaji T, Sawada $\mathrm{N}$, Goto A, et al. Inclusion of a genetic risk score into a validated risk prediction model for colorectal cancer in Japanese men improves performance. Cancer Prev Res (Phila). 2017;10: $535-41$.

11. Kirkegaard H, Johnsen NF, Christensen J, Frederiksen K, Overvad K, Tjonneland A. Association of adherence to lifestyle recommendations and risk of colorectal cancer: a prospective Danish cohort study. BMJ. 2010;341:c5504.

12. Platz EA, Willett WC, Colditz GA, Rimm EB, Spiegelman D, Giovannucci E. Proportion of colon cancer risk that might be preventable in a cohort of middle-aged US men. Cancer Causes Control. 2000;11:579-88.

13. Wei EK, Colditz GA, Giovannucci EL, Fuchs CS, Rosner BA. Cumulative risk of colon cancer up to age 70 years by risk factor status using data from the Nurses' Health Study. Am J Epidemiol. 2009;170:863-72.

14. Driver JA, Gaziano JM, Gelber RP, Lee IM, Buring JE, Kurth T. Development of a risk score for colorectal cancer in men. Am J Med. 2007;120:257-63.

15. Ahn Y, Kwon E, Shim JE, Park MK, Joo Y, Kimm K, et al. Val- idation and reproducibility of food frequency questionnaire for Korean genome epidemiologic study. Eur J Clin Nutr. 2007;61:1435-41.

16. Cho YA, Lee J, Oh JH, Shin A, Kim J. Dietary inflammatory index and risk of colorectal cancer: a case-control study in Korea. Nutrients. 2016;8:E469.

17. Shivappa N, Steck SE, Hurley TG, Hussey JR, Hebert JR. Designing and developing a literature-derived, populationbased dietary inflammatory index. Public Health Nutr. 2014; 17:1689-96.

18. Song N, Shin A, Park JW, Kim J, Oh JH. Common risk variants for colorectal cancer: an evaluation of associations with age at cancer onset. Sci Rep. 2017;7:40644.

19. Brautbar A, Pompeii LA, Dehghan A, Ngwa JS, Nambi V, Virani SS, et al. A genetic risk score based on direct associations with coronary heart disease improves coronary heart disease risk prediction in the Atherosclerosis Risk in Communities (ARIC), but not in the Rotterdam and Framingham Offspring, Studies. Atherosclerosis. 2012;223:421-6.

20. Stevens J, Juhaeri, Cai J. Changes in body mass index prior to baseline among participants who are ill or who die during the early years of follow-up. Am J Epidemiol. 2001;153:946-53.

21. Liang PS, Chen TY, Giovannucci E. Cigarette smoking and colorectal cancer incidence and mortality: systematic review and meta-analysis. Int J Cancer. 2009;124:2406-15.

22. Dunlop MG, Tenesa A, Farrington SM, Ballereau S, Brewster $\mathrm{DH}$, Koessler T, et al. Cumulative impact of common genetic variants and other risk factors on colorectal cancer risk in 42,103 individuals. Gut. 2013;62:871-81.

23. Anand P, Kunnumakkara AB, Sundaram C, Harikumar KB, Tharakan ST, Lai OS, et al. Cancer is a preventable disease that requires major lifestyle changes. Pharm Res. 2008;25:2097-116.

24. Bardou M, Barkun AN, Martel M. Obesity and colorectal cancer. Gut. 2013;62:933-47.

25. Shiels MS, Katki HA, Freedman ND, Purdue MP, Wentzensen $\mathrm{N}$, Trabert B, et al. Cigarette smoking and variations in systemic immune and inflammation markers. J Natl Cancer Inst. 2014;106:dju294.

26. Bufill JA. Colorectal cancer: evidence for distinct genetic categories based on proximal or distal tumor location. Ann Intern Med. 1990;113:779-88.

27. Iacopetta B. Are there two sides to colorectal cancer? Int J Cancer. 2002;101:403-8.

28. Shin A, Joo J, Bak J, Yang HR, Kim J, Park S, et al. Site-specific risk factors for colorectal cancer in a Korean population. PLoS One. 2011;6:e23196.

29. Wei EK, Giovannucci E, Wu K, Rosner B, Fuchs CS, Willett WC, et al. Comparison of risk factors for colon and rectal cancer. Int J Cancer. 2004;108:433-42.

30. Yeoh KG, Ho KY, Chiu HM, Zhu F, Ching JY, Wu DC, et al. The Asia-Pacific Colorectal Screening score: a validated tool that stratifies risk for colorectal advanced neoplasia in asymptomatic Asian subjects. Gut. 2011;60:1236-41. 